\title{
Rare Paraneoplastic Opsoclonus Myoclonus Syndrome (POMS) in a Recurrent Breast Cancer Patient: Importance of Early Recognition of POMS and Initiation of Rehabilitation Intervention
}

\author{
Saw Hay Mar, Tan Pei Ling, Tan YeowLeng \\ Department of Rehabilitation Medicine, Singapore General Hospital, Singapore Health Services, Outram Road, Singapore.
}

\section{Corresponding Author:}

Dr. Saw Hay Mar

Email: saw.hay.mar@singhealth.com.sg

This is an Open Access article distributed under the terms of the Creative Commons Attribution License (creativecommons.org/ licenses/by/3.0).

Received Accepted

Published

February 2, 2017

March 17, 2017

March 30, 2017

\begin{abstract}
Background: Opsoclonus myoclonus syndrome is a rare condition characterized by the amalgamation of multidirectional saccades of eye movements, involuntary myoclonus predominantly affecting trunk and limbs and cerebellar ataxia. Typical malignancies associated with paraneoplatic opsoclonus myoclonus syndrome (POMS) in adults are small cell lung carcinoma and breast cancer with variable outcomes. Case Report: The authors report here a patient with stage IV recurrent right breast cancer, presenting to the hospital with unsteady gait, jerky movements of the limbs and body. She was clinically diagnosed with POMS with positive cerebrospinal fluid anti-Ri antibody. Medical treatment with intravenous methylprednisolone and early initiation of rehabilitation improved her balance, coordination and ataxia. This led to an overall improvement in her physical function. Conclusion: This case illustrates the importance of early recognition, prompt treatment of POMS and initiation of rehabilitation in achieving better functional outcomes.
\end{abstract}

Keywords: Ataxia, Breast Neoplasms, Gait Disorders, Opsoclonus-Myoclonus Syndrome, Small Cell Lung Carcinoma.

\section{Introduction}

Paraneoplastic neurologic syndromes (PNS) are rare neurological complications affecting up to $1-3 \%$ of patients with malignancy. PNS is commonly caused by immune-mediated mechanisms triggered by an underlying tumor and it is critical for clinicians to distinguish PNS from other neurological symptoms related to metastasis, infection, ischemia, metabolic disturbances, or treatment associated toxicities [13]. PNS has been reported in association with breast cancer since late 1960s and clinical presentation of PNS can be catastrophic. It may emerge before malignancy has been identified or it may be a hint of cancer recurrence. Breast cancer related PNS encompasses several neurological disorders, such as sensory motor neuropathies, autonomic neuropathy, neuromuscular junction disorders, paraneoplastic cerebellar degeneration, opsoclonus myoclonus syndrome, stiff person syndrome, encephalomyelitis including limbic encephalopathy and paraneoplastic retinopathy [2-4]. Among those syndromes, paraneoplastic opsoclonus myoclonus syndrome (POMS) associated with breast cancer is scarce and it has been mainly reported on case series or case reports in literature [4].

This report describes a woman with recurrent breast cancer presenting a classical POMS and treatment outcome. At present, there are no POMS reports in South East Asia. Through this case, we highlight the promising outcome as a result of instantaneous medical treatment and early inpatient rehabilitation. 


\section{Case Report}

A 48-year old Chinese female with stage IV recurrent breast cancer with contralateral axillary nodal metastases on maintenance hormonal therapy with monoclonal antibody, presented to oncology unit in late 2016 due to unsteady gait and jerky movements in limbs and body by minimal tactile and audio stimuli over 3 weeks duration. As a result, she had frequent falls and was not able to ambulate and self-care independently. There was neither back pain nor sensory symptoms. Her past medical history revealed a locally advanced right breast cancer (ER positive, PR and HER-2 negative) diagnosed in 2013 for which she received neo-adjuvant chemotherapy, wide excision of right breast followed by radiation therapy and tamoxifen. One year later, she undertook right total mastectomy followed by adjuvant chemotherapy and monoclonal antibody for locally invasive recurrent ductal carcinoma in-situ. In early 2016, there was a contralateral axillary nodal metastasis and thus she had left mastectomy, axillary clearance and followed by hormonal therapy.

On clinical assessment, involuntary arrhythmic multidirectional saccades in both eyes and multi-directional nystagmus which were worse on downward gaze were noted. She had bilateral slow frequency proximal myoclonic jerks in all limbs, cerebellar signs of bilateral dysdiadochokinesia, dysmetria in upper limbs, positive heel shin test on lower limbs, truncal and gait ataxia. These features resulted in fine motor impairment, poor dynamic sitting and standing balance. Muscle strength, sensation, reflexes were normal. Proprioception was intact with negative Babinski sign. Cognitive function was intact with Montreal cognitive assessment (MOCA) 26 out of 30. Initial differential diagnoses were metastatic brain tumor versus para-neoplastic disorder. The neurologist opinion and array of investigations were sought. Magnetic resonance imaging of the brain, nuclear bone scan and positron emission

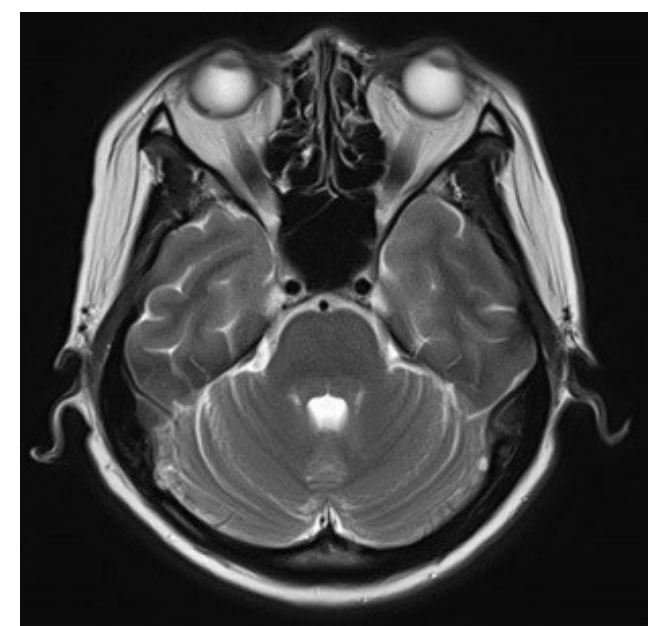

Fig.1: Axial view of T2 weighted Magnetic Resonance Imaging of the brain without metastatic lesions, ischemic changes or abnormal enhancement with gadolinium contrast.

tomography-computed tomography of thorax, abdomen, pelvic did not suggest malignancy [Fig.1]. Cerebrospinal fluid studies revealed mildly raised total protein $(0.58 \mathrm{~g} / \mathrm{L})$, other than that, it was negative for malignant cells, anti-Nmethyl-D-aspartate (NMDA) receptor antibodies, infective and toxicological panels [Table 1]. Blood investigations showed stable renal function test (serum creatinine: $132 \mu \mathrm{mol} / \mathrm{L}$, urea: $10 \mu \mathrm{mol} / \mathrm{L}$ ), normal thyroid function and liver function tests. Electroencephalogram was also negative for epileptiform activity.

A clinical diagnosis of POMS was made and she was commenced on intravenous methylprednisolone one gram per day for five days with the complete resolution of opsoclonus within a week post-steroid therapy. CSF specimen for anti-Ri antibody was subsequently reported as positive and patient was maintained on oral prednisolone $(0.5 \mathrm{mg} / \mathrm{kg}$ body weight $)$ and mycophenolate mofetil. Early rehabilitation program was initiated in rehabilitation unit as she had significant neurological and functional deficits requiring walking frame and moderate level of assistance by one person for all transfers, ambulation and Activity of Daily Livings (ADLs). 
Intensive rehabilitation program comprised of up to one hour of both physical therapy and occupational therapy per day on weekdays over three weeks duration. The program focused on range-of-motion exercises, strengthening exercises with progressive resistance training, aerobic training, truncal and balance exercises, gait retraining with assistive devices, grasp and pinch hand exercise. Speech and language therapy was arranged three times per week and it consisted of articulation and deep breathing exercises.

After a course of rehabilitation, there was improvement in dysarthria with clearer speech, ataxia and balance. Self-care, transfers and ambulation status progressed meaningfully to contact assistance from moderate assistance level. Functional independence measures (FIM) score improved from 72 upon admission (motor FIM41, cognitive FIM 31) to 88 (motor FIM 55, cognitive FIM 33) upon discharge. No more myoclonic jerks were observed.

\section{Discussion}

Paraneoplastic syndromes (PNS) are rare neurological entities and they can affect any part of central nervous system (CNS) without infiltration of cancer cells directly to the nervous system. Clinical signs and symptoms of PNS are variable depending on anatomical location of CNS and occasionally it could be a major challenge to diagnose it. Subacute cerebellar degeneration followed by limbic encephalitis and sensory neuropathy were the most frequently identified PNS of the nervous system but opsoclonus myoclonus syndrome (OMS) is a rare condition among the PNS [1,2]. Clinical presentation of OMS is characterized by the combination of rapid, involuntary, multidirectional, conjugate saccades of ocular movements without an intersaccadic interval (opsoclonus) and brief, shock-like involuntary movements of muscles (myoclonus) that predominantly involves trunk and limbs. Additional clinical features such as ataxia,
Table 1: Cerebrospinal fluid analysis.

\begin{tabular}{|l|l|l|}
\hline $\begin{array}{l}\text { Cerebrospinal fluid } \\
\text { Investigations }\end{array}$ & Result & $\begin{array}{l}\text { (Normal } \\
\text { range)/units }\end{array}$ \\
\hline $\begin{array}{l}\text { Microscopic examination } \\
\text { and cytology }\end{array}$ & & \\
Microscopic examination & Clear & \\
WBC count & 0 & cell/ $\mu \mathrm{L}$ \\
RBC count & 4 & $\mathrm{cell} / \mu \mathrm{L}$ \\
Glucose & 4.9 & $\mathrm{mmol} / \mathrm{L}$ \\
Protein (total) & 0.58 & $(0.1-0.4) \mathrm{g} / \mathrm{L}$ \\
Lactate & 2.9 & $\mathrm{mmol} / \mathrm{L}$ \\
Cytology & No malignant cells seen; & \\
& lymphocytosis & \\
Oligoclonal bands & Detected & \\
\hline Microbiological & & \\
investigations & & \\
Gram stain & No organisms seen; & \\
Bacterial culture & nil polymorphs seen & \\
AFB smear & No bacterial growth & \\
AFB culture & No AFB seen & \\
No growth of AFB after & \\
Tetraplex PCR & 6 and 8 weeks of & \\
(HSV, CMV, VZV, T. gondii) & incubation in liquid and & \\
\hline Autoimmune panel & Nolid medium, respectively & \\
GAD antibody & & \\
NMDAR antibody, IFT & Not detected & \\
LGI1 antibody \\
CASPR2 antibody & Not detected & \\
AMPAR 1\&2 antibodies & Not detected & Not detected \\
DPPX antibody & Not detected & \\
Anti-Ri antibody & Detected & \\
\hline
\end{tabular}

$\mathrm{HSV}=$ Herpes simplex virus; $\mathrm{CMV}=$ Cytomegalovirus; $\mathrm{VZV}=$ Varicella zoster virus; T.gondii $=$ Toxoplasma gondii; $\mathrm{AFB}=$ acid-fast bacilli; GAD $=$ Glutamic acid decarboxylase; NMDAR $=$ N-methyl-D-aspartate receptor; IFT = immunofluorescence test; LGI1 = leucine-rich, glioma inactivated 1; $\mathrm{CASPR}=$ contactin-associated protein AMPAR $=$ Anti-glutamate receptor; DPPX $=$ Dipeptidyl-peptidase-like protein-6; cell/ $/ \mathrm{LL}=$ number of cells per microliter; $\mathrm{g} / \mathrm{L}=$ grams per liter; $\mathrm{U} / \mathrm{mL}=$ units per milliliter; $\mathrm{mmol} / \mathrm{L}=$ millimoles per liter.

tremors, dysarthria and psychiatric symptoms are usually observed in OMS [1,5]. In adults, patients with OMS can be paraneoplastic or idiopathic, the former conveys worse prognosis than the latter [5]. Typical malignancies associated with POMS in adults are small cell lung carcinoma and breast cancer whereas neuroblastoma in pediatric population $[1,3]$. Moreover, other types of cancer such as ovarian teratoma, testicular seminoma, gastric, thymus, kidney and oropharyngeal carcinoma have been identified in association with POMS [5]. The pathophysiology of POMS 
remains ambiguous. Immune mediated mechanism may play an important role where detection of antineuronal antibodies and neuronal surface antigens in serum and CSF of the patients were evident. Most of the POMS may not be associated with well characterized antibodies with the exception of breast carcinoma which is usually associated with anti-Ri antibodies $[3,4,5,7]$. Literatures have supported that disruption of the tonic inhibitory control of saccadic neurons by the omnipause neurons in the pontine reticular formation could be a possible cause of opsoclonus. Nonetheless, the omnipause neurons at autopsy may reveal completely normal brain $[2,3]$.

\section{Patients with POMS are generally} treated with immunotherapy, treatment of underlying malignancy and symptomatic treatment. Immunotherapy includes intravenous immunoglobulin, corticosteroids, immunesuppressors, plasmapheresiswith variable outcomes $[2,4,6,10]$. Cancer rehabilitation is recently recognized as an integral part of continuum of care in cancer management and emerging evidences support that rehabilitation improves physical functioning, strength, psychosocial health outcome, quality of life and immune function $[8,9]$. At present, only limited information is availablefor rehabilitation outcomes of PNS population although numerous published literatures are available for its pathophysiology, medical treatment and prognosis $[11,12]$. This case report intends to appreciate early awareness of classical presentation of POMS, better functional outcome with comprehensive rehabilitation in collaboration with prompt medical treatment.

In our patient, the clinical presentation initially alerted to medical team as high possibility of metastatic disease in view of underlying history of recurrent carcinoma of breast. However, neuro-diagnostic imaging and CSF findings were not consistent with metastatic disease. As such, diagnosis was favored towards paraneoplastic syndrome. She was commenced on early immunosuppressant treatment with improvement in total and rapid resolution of opsoclonus and myoclonic jerks in her limbs. This report highlighted timely diagnosis of POMS and initiation of treatment is critical which can potentially relieve the debilitating symptoms. Upon admission to inpatient rehabilitation unit, this patient had some significant impairments, namely, myoclonus, truncal, axial and gait ataxia resulted her in requiring moderate level of assistance for activity of daily livings and mobility. Medical therapy together with timely commencement of rehabilitation intervention seemed to improve functional outcome in this case.

\section{Conclusion}

This case illustrates the need to recognize clinical presentation of rare POMS and importance of early rehabilitation intervention. We believe that exploring more studies on rehabilitation outcome in POMS are required to support our findings.

Contributors: SHM: Manuscript writing and literature review; TPL, TYL: Manuscript revision and literature review. All authors were involved in case management. SHM will act as guarantor. All authors approved the final version of the manuscript.

Funding: None; Competing interests: None stated.

\section{References}

1. Samuel M, Flavia DA, Emmanuelle L, Sandrine L, Giovanna S. Paraneoplastic neurologic syndrome: Clinical presentation and management. Curr Probl Cancer. 2014;38:115-134.

2. Robert BD, Jerome BP. Paraneoplastic syndromes involving in the nervous system. $N$ Engl $J$ Med. 2003;349:1543-1554.

3. Gatti G, Simsek S, Kurne A,Zurrida S, Naninato P, Veronesi $\mathrm{P}$, et al. Paraneoplastic neurological disorders in breast cancer. The Breast. 2003;12:203-207.

4. Ibrahim F, Patrick D. Paraneoplastic neurological complications of breast cancer. Exp Hematol Oncol. 2016;5:29.

5. Armangué T, Sabater L, Torres-Vega E, MartínezHernández E, Ariño H, Petit-Pedrol M, et al. Clinical and Immunological Features of Opsoclonus-Myoclonus 
Syndrome in the Era of Neuronal Cell Surface Antibodies. JAMA Neurol. 2016;73:417-424.

6. Klaas JP, Ahlskog JE, Pittock SJ, Matsumoto JY, Aksamit AJ, Bartleson JD, et al. Adult onset Opsoclonusmyoclonus syndrome. Arch Neurol. 2012;69:1598-1607.

7. Jessica P, Josep D. Movement disorders in paraneoplastic and autoimmune disease. Curr Opin Neurol. 2011;24: 346-353.

8. Rosalind RS, Kristiann CH, Wendy JB. Exercise and cancer rehabilitation: A systematic review. Cancer Treatment Reviews. 2010;36:185-194.

9. Silver JK, Baima J. Cancer Prehabilitation: An opportunity to decrease treatment related morbidity, increase cancer treatment options, and improve physical and psychological health outcomes. Am J Phys Med Rehabil. 2013;82:715-727.

10. Bataller L, Graus F, Saiz A, Vilchez JJ. Clinical outcome in adult onset idiopathic or paraneoplastic opsoclonus myoclonus. Brain. 2001;124;437-443.

11. Sean RS, Jack BF. Paraneoplastic stiff person syndrome: Inpatient rehabilitation outcome of a rare disease from two cancer rehabilitation program. J Rehabil Med. 2016;48:639-642.

12. Fu JB, Raj VS, Asher A, Lee J, Guo Y, Konzen BS, Bruera $\mathrm{E}$, et al. Inpatient rehabilitation performance of patients with paraneoplastic cerebellar degeneration. Arch Phys Med Rehabil. 2014;95:2496-2499. 